\title{
Doença renal crônica: influencia sistêmica na odontologia e manifestações bucais
}

\author{
Chronic kidney disease: systemic influence on dentistry and oral manifestations \\ Enfermedad renal crónica: influencia sistémica en la odontología y las manifestaciones orales
}

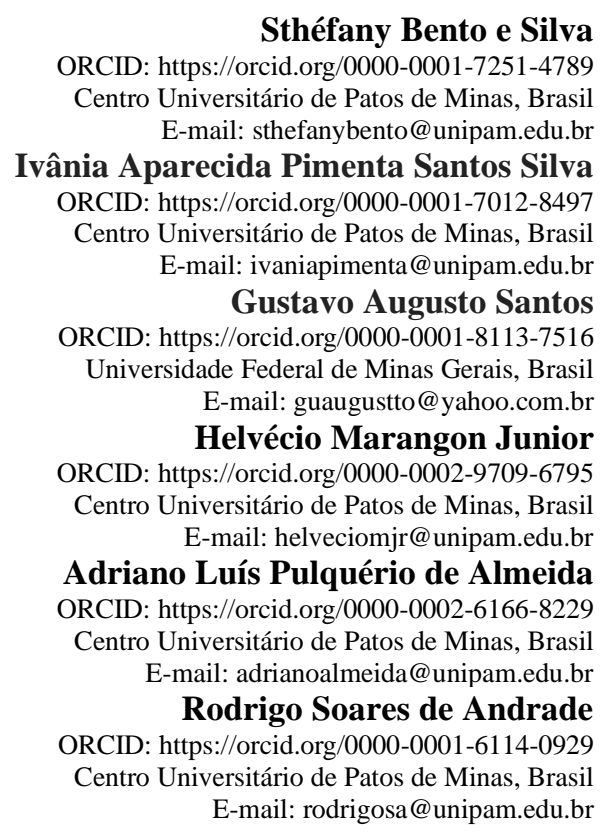

\section{Resumo}

A doença renal crônica é uma doença que teve sua prevalência aumentada nos últimos temos. É importante lembrar que os pacientes que possuem doença renal crônica (DRC) apresentam manifestações orais oriundas de influencia sistêmica, logo o objetivo desse trabalho é estabelecer as principais manifestações bucais que acometem os pacientes com insuficiência renal crônica. Trata-se de um estudo do tipo bibliográfico, básico, com análise integrativa, para o qual foram utilizadas as bases de dados Pubmed, Google Schoolar e Scielo, incluídos artigos dos anos 2011 a 2021 , além de artigos clássicos, onde foram pesquisados os seguintes descritores: "Dental care"; "Dentistry"; "Chronic renal failure"; "Chronic kidney disease"; "Kidney failure"; "Renal insufficiency chronic", além do descritor booleano "AND”. A literatura possui uma vasta informação sobre essas manifestações bucais que incluem: cárie dentária, perda de inserção clínica, doença periodontal, fluorose, cálculo dentário, placa bacteriana, inflamação perirradicular, infecção microbiana no sistema de canais radiculares, disgeusia, hálito urêmico, xerostomia, hipoplasia de esmalte e distúrbios gustativos. Diante a todas as informações é importante ressaltar a importância do acompanhamento e assistência do cirurgião dentista a esses pacientes.

Palavras-chave: Insuficiência renal crônica; Assistência odontológica para doentes crônicos; Manifestações bucais.

\begin{abstract}
Chronic kidney disease is a disease that has had its prevalence increased in recent years. It is important to remember that patients with chronic kidney disease (CKD) have oral manifestations arising from systemic influence, so the aim of this work is to establish the main oral manifestations that affect patients with chronic renal failure. This is a bibliographic study, basic, with integrative analysis, for which the databases Pubmed, Google Schoolar and Scielo were used, including articles from 2011 to 2021, in addition to classic articles, where the following descriptors were searched: "Dental care"; "Dentistry"; "Chronic renal failure"; "Chronic kidney disease"; "Kidney failure"; "Chronic renal insufficiency", and the Boolean descriptor "AND". The literature has vast information about these oral manifestations that include: dental caries, clinical attachment loss, periodontal disease, fluorosis, dental calculus, dental plaque, peri-radicular inflammation, microbial infection in the root canal system, dysgeusia, uremic breath, xerostomia, enamel hypoplasia, and taste disorders. In view of all this information, it is important to emphasize the importance of the dental surgeon's follow-up and assistance to these patients.
\end{abstract}

Keywords: Renal insufficiency, chronic; Dental care for chronically III; Oral manifestations. 


\begin{abstract}
Resumen
La enfermedad renal crónica es una enfermedad cuya prevalencia ha aumentado en los últimos años. Es importante tener en cuenta que los pacientes que padecen una enfermedad renal crónica (DRC) presentan manifestaciones orales oriundas de la influencia sistémica, por lo que el objetivo de este trabajo es establecer las principales manifestaciones bucales que presentan los pacientes con insuficiencia renal crónica. Se trata de un estudio bibliográfico, básico, con análisis integrador, para el que se utilizaron las bases de datos Pubmed, Google Schoolar y Scielo, incluyendo artículos desde 2011 hasta 2021, además de artículos clásicos, donde se buscaron los siguientes descriptores: "Dental care"; "Dentistry"; "Chronic renal failure"; "Chronic kidney disease"; "Kidney failure"; "Chronic renal insufficiency", además del descriptor booleano "AND". La literatura tiene una amplia información sobre estas manifestaciones orales que incluyen: caries dental, pérdida de inserción clínica, enfermedad periodontal, fluorosis, cálculo dental, placa bacteriana, inflamación perirradicular, infección microbiana en el sistema de canales radiculares, disgeusia, aliento urémico, xerostomía, hipoplasia del esmalte y trastornos del gusto. Ante toda esta información, es importante destacar la importancia del seguimiento y la asistencia del cirujano dental a estos pacientes.
\end{abstract}

Palabras clave: Insuficiencia renal crónica; Atención dental para enfermos crónicos; Manifestaciones bucales.

\title{
1. Introdução
}

Os rins são órgãos complexos, de alta relevância e funções, que controlam o volume de fluidos, além da produção e secreção de hormônios como a eritropoietina, vitamina D e renina (Xie et al, 2014; Ausavarungnirun et al., 2016). A forma de controle dos líquidos acontece, de modo que o excesso de água do corpo seja eliminado pela urina, o chamado efeito diurético. O rim exerce controle sobre os sais em nosso corpo, eliminando seus excessos ou guardando-os em situações de necessidade. (Ausavarungnirun et al., 2016; Sharma et al., 2020)

A doença renal crônica (DRC) é uma enfermidade com alta prevalência, sendo então, uma adversidade médica. A DRC possui uma incidência elevada que é dificultada pelo registro de um diagnostico incorreto, além da alta taxa de mortalidade sobre os quais a comunidade científica internacional tem se preocupado nas últimas duas décadas. (Xie et al., 2014; Huang et al., 2017; Sharma et al., 2020)

A insuficiência renal crônica (IRC) pode causar várias modificações sistêmicas, gastrointestinais, cardiovasculares, neurológicas, eletrolíticas e metabólicas ósseas (Xie et al., 2014; Ausavarungnirun et al., 2016). Na odontologia, é importante entender que os pacientes com DRC apresentam um número significativo de manifestações orais que não são absolutamente patognomônicas da doença e não são essenciais para o seu diagnóstico, e sim consequência de sua presença (Ausavarungnirun et al., 2016; Huang et al., 2017).

Alguns estudos, como o de Swapna et al., (2017) expôs que cerca de $90 \%$ dos pacientes com insuficiência renal crônica têm algum tipo de manifestação bucal devido aos efeitos colaterais1w do tratamento e drogas utilizadas, como resultado de uma série de desequilíbrios urêmicos, metabólicos, endocrinológicos e imunológicos (Andrade et al., 2015; Swapna; Koppolu; Prince, 2017).

O manejo odontológico de pacientes em diálise pode ser complexo, dada a prevalência de comorbidades como diabetes, hipertensão, osteoartrite renal e imunossupressão, presença de próteses cardíacas e uso de anti-hipertensivos, anticoagulantes ou antiplaquetários (Xie et al., 2014; Swapna; Ausavarungnirun et al., 2016; Koppolu; Prince, 2017; Marinosk et al., 2019). Esses pacientes estão propensos a uma variedade de problemas, como doença periodontal, redução da câmara pulpar, anormalidades do esmalte, perda prematura dos dentes e xerostomia. $\mathrm{O}$ atendimento odontológico, assim como as medidas preventivas primárias, tem sido negligenciado nesses pacientes. (Sturgill et al., 2016; Swapna; Koppolu; Prince, 2017)

Desse modo, cirurgiões dentistas enfrentam certa dificuldade em relação aos atendimentos, principalmente por não possuírem um protocolo pré-estabelecido que os guie (Sturgill et al., 2016). O objetivo desse trabalho é estabelecer as principais manifestações bucais que acometem os pacientes com doença renal crônica, que devem ser observadas pelo cirurgião dentista. 


\section{Metodologia}

Esse trabalho trata-se de um estudo do tipo bibliográfico, básico, com análise integrativa, no qual foi realizado para descrever as alterações presentes no paciente renal crônico que afetam o tratamento odontológico. (Estrela, 2018).

A busca literária foi feita utilizando a base de dados Pubmed (MEDLINE), Google Schoolar e Scielo, dos anos de 2011 a 2021, pesquisado os seguintes descritores: "Dental Care”, "Dentistry”, “Chronic Renal Failure”, “Chronic Kidney Disease", "Kidney Failure", "Renal Insufficiency Chronic”, além do descritor booleano “AND”.

Foram incluídos apenas artigos dos últimos 10 anos (2011 a 2021), possuir textos completos, ter relação entre doenças bucais ou atendimento odontológico aos pacientes renais. Dessa forma, foram excluídos artigos que se tratavam de revisões de literatura (narrativa, integrativa ou sistemática), não possuir relação com o tema proposto e artigos fora do intervalo de tempo definido

\section{Resultados}

A pesquisa foi feita a partir dos dados coletados na literatura, encontrados nos sites de busca como PubMed e Google Schoolar. O fluxograma (Figura 1), apresenta como foi feita a seleção desses artigos.

Os artigos coletados foram tabelados (Tabela 1) levando em consideração o nome do autor principal e ano de publicação, jornal, revista ou periódico onde foi publicado, país onde foi realizado, tipo de artigo, manifestações bucais apresentadas e protocolo de tratamento.

Desses artigos, a doença periodontal se apresentou como o sintoma mais comum entre os pacientes, em cerca de 18 artigos dos 28 utilizados. A doença cárie e a xerostomia também se destacaram como sintomas frequentes, em aproximadamente 10 artigos do total.

A presença de cálculo dental e a placa bacteriana estão associados a má higienização bucal, esses sintomas tem sido relatados com frequência em pacientes com DRC. Juntamente aos fatores sistêmicos, esses pacientes têm desenvolvido a disgeusia e o hálito urêmico, devido o aumento de uréia salivar, podendo causar também distúrbios gustativos.

A maioria dos autores não propôs um protocolo concreto para o atendimento desses pacientes, mas destaca-se a importância do acompanhamento do cirurgião dentista durante o tratamento, além de consultas e ações preventivas. 
Figura 1: Fluxograma do processo de busca na literatura.

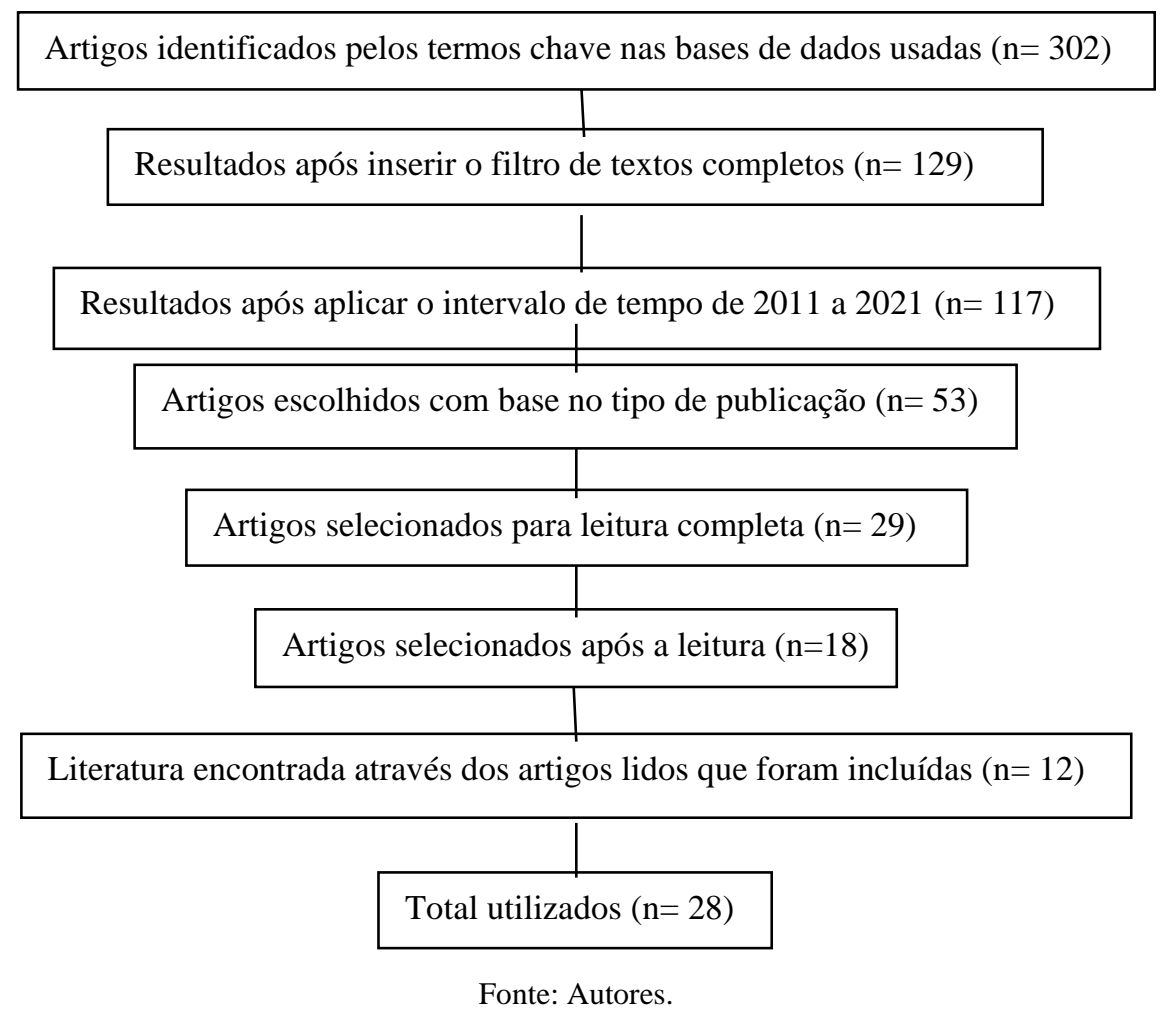

Tabela 1: Artigos utilizados na revisão integrativa sobre o atendimento odontológico ao paciente portador de doença renal crônica, mostrando o periódico publicado, país de origem, amostra utilizada, achados bucais ou tratamentos odontológicos feitos e o protocolo proposto por cada autor.

\begin{tabular}{|c|c|c|c|c|c|}
\hline $\begin{array}{c}\text { Autor } \\
\text { principal/ Ano }\end{array}$ & Periódico & $\begin{array}{l}\text { Pais de } \\
\text { origem }\end{array}$ & Amostra & $\begin{array}{c}\text { Achados bucais/ } \\
\text { Tratamento } \\
\text { odontológico }\end{array}$ & Protocolo proposto \\
\hline $\begin{array}{l}\text { Bhatsange; } \\
\text { Patil, 2012 }\end{array}$ & $\begin{array}{l}\text { Journal of indian society } \\
\text { of periodontology }\end{array}$ & Índia & $\begin{array}{c}\text { Estudo transversal } \\
\text { descritivo/ amostra de } 75 \\
\text { pacientes com DRC e } 25 \\
\text { no grupo controle }\end{array}$ & $\begin{array}{l}\text { Uréia salivar, doença } \\
\text { periodontal, higiene oral } \\
\text { deficiente, perda de } \\
\text { inserção }\end{array}$ & $\begin{array}{l}\text { Remoção de fontes de } \\
\text { infecção e } \\
\text { acompanhamento } \\
\text { odontológico }\end{array}$ \\
\hline $\begin{array}{l}\text { Martins et al., } \\
2012\end{array}$ & $\begin{array}{l}\text { Pediatric nephrology } \\
\text { (Berlin, Germany) }\end{array}$ & Brasil & $\begin{array}{c}\text { Estudo caso-controle/ } \\
\text { Amostra de } 30 \text { pacientes } \\
\text { com DRC e } 30 \text { no grupo } \\
\text { controle }\end{array}$ & $\begin{array}{l}\text { Placa bacteriana, } \\
\text { inflamação gengival, } \\
\text { cálculo }\end{array}$ & $\begin{array}{l}\text { Instrução de higienização } \\
\text { bucal, controle dietético }\end{array}$ \\
\hline $\begin{array}{c}\text { Parkar; } \\
\text { Ajithkrishna, } \\
2012\end{array}$ & $\begin{array}{l}\text { Indian journal of } \\
\text { nephrology }\end{array}$ & Índia & $\begin{array}{c}\text { Estudo transversal/ } \\
\text { amostra de } 152 \text { pacientes } \\
\text { com IRC e } 152 \text { no grupo } \\
\text { controle }\end{array}$ & $\begin{array}{l}\text { Doença periodontal, } \\
\text { cálculo, placa bacteriana }\end{array}$ & $\begin{array}{c}\text { Atendimento } \\
\text { odontológico precoce e } \\
\text { profilaxia }\end{array}$ \\
\hline $\begin{array}{l}\text { Sekiguchi et } \\
\text { al., } 2012\end{array}$ & $\begin{array}{l}\text { Special care in dentistry: } \\
\text { official publication of } \\
\text { the American } \\
\text { Association of Hospital } \\
\text { Dentists, the Academy } \\
\text { of Dentistry for the } \\
\text { Handicapped, and the } \\
\text { American Society for } \\
\text { Geriatric Dentistry }\end{array}$ & Brasil & $\begin{array}{c}\text { Estudo transversal e } \\
\text { caso-controle/ Amostra } \\
\text { de } 94 \text { pacientes com } \\
\text { DRC }\end{array}$ & $\begin{array}{l}\text { Perda de inserção, } \\
\text { doença periodontal }\end{array}$ & Não se aplica \\
\hline $\begin{array}{c}\text { Tiwari et al., } \\
2013\end{array}$ & Journal of Renal Care & Índia & $\begin{array}{c}\text { Estudo de caso-controle/ } \\
\text { amostra e } 30 \text { pacientes } \\
\text { com DRC e } 30 \text { no grupo } \\
\text { controle }\end{array}$ & $\begin{array}{c}\text { Doença periodontal, } \\
\text { cálculo, xerostomia, } \\
\text { halitose }\end{array}$ & $\begin{array}{l}\text { Hidratação contínua da } \\
\text { boca e desbridamento de } \\
\text { dentes e gengivas, } \\
\text { instrução de higiene oral } \\
\text { e manutenção da saúde } \\
\text { bucal }\end{array}$ \\
\hline
\end{tabular}




\begin{tabular}{|c|c|c|c|c|c|}
\hline $\begin{array}{l}\text { Gautam et al., } \\
2014\end{array}$ & $\begin{array}{l}\text { Journal of International } \\
\text { Society of Preventive \& } \\
\text { Community Dentistry }\end{array}$ & Índia & $\begin{array}{c}\text { Estudo transversal/ } \\
\text { amostra de } 206 \text { pacientes } \\
\text { com DRC em estágio } \\
\text { terminal }\end{array}$ & $\begin{array}{l}\text { Cárie, macroglossia, } \\
\text { candidíase, doença } \\
\text { periodontal, líquen } \\
\text { plano, ulcerações, } \\
\text { melanose de fumante, } \\
\text { estomatite urêmica }\end{array}$ & $\begin{array}{l}\text { Prevenção odontológica } \\
\text { e conscientização da } \\
\text { importância da saúde } \\
\text { bucal }\end{array}$ \\
\hline $\begin{array}{l}\text { Tadakamadla } \\
\text { et al., } 2014\end{array}$ & $\begin{array}{l}\text { Special care in dentistry: } \\
\text { official publication of } \\
\text { the American } \\
\text { Association of Hospital } \\
\text { Dentists, the Academy } \\
\text { of Dentistry for the } \\
\text { Handicapped, and the } \\
\text { American Society for } \\
\text { Geriatric Dentistry }\end{array}$ & Índia & $\begin{array}{c}\text { Amostra de } 74 \text { pacientes } \\
\text { com DRC e } 150 \text { no grupo } \\
\text { controle }\end{array}$ & $\begin{array}{c}\text { Baixa prevalência de } \\
\text { cárie, cálculo, higiene } \\
\text { oral deficiente, doença } \\
\text { periodontal }\end{array}$ & Não se aplica \\
\hline Xie et al., 2014 & $\begin{array}{c}\text { Hemodialysis } \\
\text { international. } \\
\text { International } \\
\text { Symposium on Home } \\
\text { Hemodialysis }\end{array}$ & China & $\begin{array}{c}\text { Amostra de } 306 \\
\text { pacientes com DRC }\end{array}$ & $\begin{array}{l}\text { Sangramento gengival, } \\
\text { dor dental aguda }\end{array}$ & $\begin{array}{l}\text { Manutenção da saúde } \\
\text { bucal }\end{array}$ \\
\hline $\begin{array}{l}\text { Andrade et al., } \\
\qquad 2015\end{array}$ & $\begin{array}{c}\text { Clinical oral } \\
\text { investigations }\end{array}$ & Brasil & $\begin{array}{c}\text { Amostra de } 52 \text { pacientes } \\
\text { com DRC }\end{array}$ & $\begin{array}{l}\text { Menor prevalência de } \\
\text { cárie, placa bacteriana, } \\
\text { inflamação gengival, } \\
\text { cálculo, uréia salivar }\end{array}$ & $\begin{array}{c}\text { Tratamento periodontal, } \\
\text { controle de placa } \\
\text { bacteriana }\end{array}$ \\
\hline $\begin{array}{l}\text { Araujo et al., } \\
2015\end{array}$ & BMC Nephrology & EUA & $\begin{array}{l}\text { Estudo piloto transversal/ } \\
\text { amostra de } 14 \text { pacientes } \\
\text { com DRC e } 13 \text { no grupo } \\
\text { controle }\end{array}$ & Doença periodontal & Não se aplica \\
\hline $\begin{array}{l}\text { Fregoneze et } \\
\text { al., } 2015\end{array}$ & $\begin{array}{l}\text { Special care in dentistry: } \\
\text { official publication of } \\
\text { the American } \\
\text { Association of Hospital } \\
\text { Dentists, the Academy } \\
\text { of Dentistry for the } \\
\text { Handicapped, and the } \\
\text { American Society for } \\
\text { Geriatric Dentistry }\end{array}$ & Brasil & $\begin{array}{l}\text { Estudo transversal e } \\
\text { caso-controle/ Amostra } \\
\text { com } 34 \text { pacientes com } \\
\text { DRC e } 34 \text { no grupo } \\
\text { controle }\end{array}$ & $\begin{array}{l}\text { Placa bacteriana, baixa } \\
\text { prevalência de cáries }\end{array}$ & $\begin{array}{l}\text { Tratamento preventivo, } \\
\text { ortodôntico }\end{array}$ \\
\hline $\begin{array}{l}\text { Palmer et al., } \\
2015\end{array}$ & $\begin{array}{l}\text { American journal of } \\
\text { kidney diseases: the } \\
\text { official journal of the } \\
\text { National Kidney } \\
\text { Foundation }\end{array}$ & $\begin{array}{l}\text { Italia, } \\
\text { Espanha, } \\
\text { França, } \\
\text { Polônia, } \\
\text { Portugal, } \\
\text { Hungria E } \\
\text { Argentina }\end{array}$ & $\begin{array}{l}\text { Estudo de Coorte } \\
\text { Multinacional/ amostra } \\
\text { de } 4.205 \text { pacientes com } \\
\text { DRC em estágio terminal }\end{array}$ & $\begin{array}{l}\text { Cárie, dentes ausentes, } \\
\text { higiene oral deficiente }\end{array}$ & $\begin{array}{l}\text { Uso de fio dental, visitas } \\
\text { frequentes ao dentista, } \\
\text { trocas de escova de } \\
\text { dente, orientação de } \\
\text { higiene oral, tratamento } \\
\text { não cirúrgico periodontal }\end{array}$ \\
\hline $\begin{array}{l}\text { Schmalz et al., } \\
2016\end{array}$ & BMC Oral Health & Alemanha & $\begin{array}{l}\text { Estudo clínico de seção } \\
\text { transversal/ amostra de } \\
70 \text { pacientes com DRC e } \\
\text { pós transplantados }\end{array}$ & $\begin{array}{l}\text { Hiperplasia gengival, } \\
\text { edentulismo, doença } \\
\text { periodontal, }\end{array}$ & $\begin{array}{c}\text { Tratamento periodontal, } \\
\text { tratamento preventivo } \\
\text { precoce }\end{array}$ \\
\hline $\begin{array}{l}\text { Nylund et al., } \\
2017\end{array}$ & $\begin{array}{l}\text { Clinical oral } \\
\text { investigations }\end{array}$ & Finlândia & $\begin{array}{l}\text { Estudo } \\
\text { longitudinal/amostra de } \\
53 \text { pacientes com DRC }\end{array}$ & $\begin{array}{c}\text { Herpes labial, doença } \\
\text { periodontal, xerostomia, } \\
\text { candidíase, acúmulo de } \\
\text { placa }\end{array}$ & $\begin{array}{l}\text { Erradicação de focos } \\
\text { infecciosos, uso de } \\
\text { escovas interdentais, } \\
\text { pastas de dentes } \\
\text { fluoretadas, terapia de } \\
\text { manutenção regular, } \\
\text { controle e tratamento de } \\
\text { infecção }\end{array}$ \\
\hline $\begin{array}{l}\text { Sharma., et al. } \\
2017\end{array}$ & Trials & Reino Unido & Ensaio aleatório & Doença periodontal & $\begin{array}{l}\text { Instrução de higiene oral, } \\
\text { raspagem sub e supra } \\
\text { gengival, Desbridamento } \\
\text { não cirúrgico da } \\
\text { superfície radicular, } \\
\text { terapia de manutenção } \\
\text { periodontal, } \\
\text { acompanhamento } \\
\text { odontológico }\end{array}$ \\
\hline $\begin{array}{c}\text { Swapna; } \\
\text { Koppolu; } \\
\text { Prince, 2017 }\end{array}$ & $\begin{array}{c}\text { Saudi journal of kidney } \\
\text { diseases and } \\
\text { transplantation: an } \\
\text { official publication of } \\
\text { the Saudi Center for } \\
\text { Organ Transplantation, } \\
\text { Saudi Arabia } \\
\end{array}$ & Índia & $\begin{array}{c}\text { Amostra de } 194 \\
\text { pacientes com DRC }\end{array}$ & $\begin{array}{l}\text { Xerostomia, hálito } \\
\text { urêmico, petéquias e } \\
\text { equimoses na mucosa, } \\
\text { ulceração, hipoplasia de } \\
\text { esmalte, disgeusia, } \\
\text { distúrbios gustativos }\end{array}$ & $\begin{array}{l}\text { Consultas de prevenção } \\
\text { com o cirurgião dentista }\end{array}$ \\
\hline
\end{tabular}




\begin{tabular}{|c|c|c|c|c|c|}
\hline $\begin{array}{l}\text { Nascimento et } \\
\text { al., } 2018\end{array}$ & $\begin{array}{l}\text { Revista Gaúcha de } \\
\text { Odontologia }\end{array}$ & Brasil & $\begin{array}{c}\text { Estudo transversal/ } \\
\text { Amostra de } 74 \text { pacientes } \\
\text { com DRC }\end{array}$ & $\begin{array}{l}\text { Cálculo, doença } \\
\text { periodontal, disgeusia }\end{array}$ & Não se aplica \\
\hline $\begin{array}{l}\text { Kopić et al., } \\
2019\end{array}$ & Acta clinica Croatica & Croácia & $\begin{array}{c}\text { Estudo de coorte } \\
\text { transversal/ amostra de } \\
80 \text { pacientes com DRC }\end{array}$ & Doença periodontal & $\begin{array}{l}\text { Consultas de prevenção } \\
\text { com o cirurgião dentista }\end{array}$ \\
\hline $\begin{array}{l}\text { Marinoski et } \\
\text { al., } 2019\end{array}$ & Archives of oral biology & Sérvia & $\begin{array}{l}\text { Estudo clínico de corte } \\
\text { transversal/ amostra de } \\
75 \text { pacientes com DRC e } \\
25 \text { no grupo controle }\end{array}$ & $\begin{array}{l}\text { Mucosa pálida, doença } \\
\text { periodontal, disgeusia, } \\
\text { xerostomia, hálito } \\
\text { urêmico }\end{array}$ & Não se aplica \\
\hline $\begin{array}{l}\text { Menezes et al., } \\
2019\end{array}$ & $\begin{array}{l}\text { Medicina oral, patologia } \\
\text { oral y cirugia bucal }\end{array}$ & Brasil & $\begin{array}{c}\text { Estudo caso-controle } \\
\text { aninhado a um estudo de } \\
\text { corte transversal/ amostra } \\
\text { de } 107 \text { pacientes com } \\
\text { DRC e } 107 \text { no grupo } \\
\text { controle }\end{array}$ & $\begin{array}{l}\text { Uréia salivar, baixa } \\
\text { concentração de cálcio, } \\
\text { alta contagem de } \\
\text { Streptococcus mutans }\end{array}$ & $\begin{array}{l}\text { Acompanhamento } \\
\text { regular no início da } \\
\text { diálise }\end{array}$ \\
\hline $\begin{array}{l}\text { Misaki et al., } \\
2019\end{array}$ & PloS one & Japão & $\begin{array}{c}\text { Amostra de } 80 \\
\text { pacientes DRC e } 76 \text { no } \\
\text { grupo controles }\end{array}$ & $\begin{array}{c}\text { Doença periodontal, } \\
\text { cárie, dentes perdidos, } \\
\text { xerostomia }\end{array}$ & Controle sistêmico \\
\hline Pham; le, 2019 & $\begin{array}{c}\text { International Journal of } \\
\text { Dental Hygiene }\end{array}$ & Vietnã & $\begin{array}{c}\text { Amostra de } 110 \\
\text { pacientes com DRC e } \\
109 \text { pacientes saudáveis }\end{array}$ & $\begin{array}{l}\text { Xerostomia, menor } \\
\text { prevalência de cárie }\end{array}$ & Não se aplica \\
\hline $\begin{array}{l}\text { Ruokonen et } \\
\text { al., } 2019\end{array}$ & $\begin{array}{l}\text { Clinical oral } \\
\text { investigations }\end{array}$ & Finlândia & $\begin{array}{l}\text { Estudo de coorte clínico } \\
\text { prospectivo longitudinal/ } \\
\text { amostra de } 53 \text { pacientes } \\
\text { pós transplante }\end{array}$ & $\begin{array}{l}\text { Cárie, doença } \\
\text { periodontal, cálculo, } \\
\text { disgeusia, disfagia, } \\
\text { xerostomia, infecções }\end{array}$ & Não se aplica \\
\hline $\begin{array}{l}\text { Silva et al., } \\
2019\end{array}$ & $\begin{array}{l}\text { Quality of life research: } \\
\text { an international journal } \\
\text { of quality of life aspects } \\
\text { of treatment, care and } \\
\text { rehabilitation }\end{array}$ & Brasil & $\begin{array}{c}\text { Estudo transversal/ } \\
\text { amostra de } 200 \text { pacientes } \\
\text { com DRC e } 82 \text { no grupo } \\
\text { controle }\end{array}$ & $\begin{array}{l}\text { Higiene oral deficiente, } \\
\text { cárie, inflamação } \\
\text { gengival, hipoplasia de } \\
\text { esmalte }\end{array}$ & $\begin{array}{c}\text { Consultas de promoção a } \\
\text { saúde bucal e } \\
\text { preventivas, }\end{array}$ \\
\hline $\begin{array}{c}\text { Kesmez et al., } \\
2020\end{array}$ & $\begin{array}{l}\text { APMIS: acta } \\
\text { pathologica, } \\
\text { microbiologica, et } \\
\text { immunologica } \\
\text { Scandinavica }\end{array}$ & Dinamarca & $\begin{array}{c}\text { Amostra de } 14 \text { pacientes } \\
\text { com DRC }\end{array}$ & $\begin{array}{c}\text { Xerostomia, dor na } \\
\text { mucosa oral, distúrbios } \\
\text { gustativos, higiene oral } \\
\text { deficiente, doença } \\
\text { periodontal, } \\
\text { hipossalivação, } \\
\text { candidíase, língua } \\
\text { eritematosa, atrófica e } \\
\text { fissurada, lesões de } \\
\text { líquen plano, }\end{array}$ & Instrução de higiene oral \\
\hline $\begin{array}{l}\text { Sarmento et } \\
\text { al., } 2020\end{array}$ & $\begin{array}{l}\text { Acta odontologica } \\
\text { Scandinavica }\end{array}$ & Brasil & $\begin{array}{c}\text { Estudo de coorte } \\
\text { observacional } \\
\text { prospectivo/ Amostra de } \\
80 \text { pacientes com DRC }\end{array}$ & $\begin{array}{c}\text { Xerostomia, hiperplasia } \\
\text { gengival, leucoplasia } \\
\text { pilosa, candidíase, } \\
\text { ulceras, herpesvirus } \\
\text { (HSV) }\end{array}$ & $\begin{array}{l}\text { Terapia antiviral com } \\
\text { ganciclovir }\end{array}$ \\
\hline $\begin{array}{l}\text { Sharma et al., } \\
2020\end{array}$ & $\begin{array}{c}\text { Journal of Family } \\
\text { Medicine and Primary } \\
\text { Care }\end{array}$ & Índia & $\begin{array}{c}\text { Estudo transversal/ } \\
\text { amostra de } 50 \text { pacientes } \\
\text { com DRC e } 50 \text { no grupo } \\
\text { controle }\end{array}$ & $\begin{array}{l}\text { Cárie, perda de inserção } \\
\text { clínica, doença } \\
\text { periodontal, fluorose e } \\
\text { erosão }\end{array}$ & $\begin{array}{l}\text { Check-up odontológico, } \\
\text { acompanhamento } \\
\text { durante o tratamento } \\
\text { incluindo pós transplante } \\
\text { e orientação quanto a } \\
\text { higiene bucal }\end{array}$ \\
\hline $\begin{array}{c}\text { Chiu et al., } \\
2021\end{array}$ & $\begin{array}{l}\text { International journal of } \\
\text { environmental research } \\
\text { and public health. }\end{array}$ & Japão & $\begin{array}{l}\text { Estudo de coorte } \\
\text { retrospectivo } \\
\text { observacional }\end{array}$ & $\begin{array}{l}\text { Terapia endodôntica, } \\
\text { doença periodontal, } \\
\text { inflamação } \\
\text { perirradicular, infecção } \\
\text { microbiana no sistema } \\
\text { de canais radiculares, } \\
\text { higiene oral deficiente }\end{array}$ & Não se aplica \\
\hline
\end{tabular}

DRC: doença renal crônica. Fonte: Autores.

\section{Discussão}

Com base nos resultados e nos artigos incluídos no presente trabalho, sobre as manifestações bucais que incluem: cárie, perda de inserção clínica, doença periodontal, fluorose, cálculo dental, placa bacteriana, inflamação perirradicular, infecção microbiana no sistema de canais radiculares, disgeusia, hálito urêmico, xerostomia, hipoplasia de esmalte e distúrbios 
gustativos (Parkar; Ajithkrishna, 2012; Tiwari et al., 2013; Swapna; Koppolu; Prince, 2017; Nascimento et al., 2018; Misaki et al., 2019; Sharma et al., 2020; Chiu et al., 2021).

O não funcionamento dos rins pode refletir de forma negativa em todos os sistemas do corpo. Pensando dessa forma, a cavidade bucal também pode ser atingida em cerca de $90 \%$ dos casos (Xie et al., 2014). A DRC é capaz de predispor alterações bucais tanto em tecido mole quanto tecido duro (Andrade et al., 2015; Silva et al., 2019).

Sendo a principal manifestação bucal, a doença periodontal, encontrada em 35\% dos artigos analisados, ocorre devido à piora de alguns fatores como a desordem endocrinológica e do metabolismo ósseo, que não podem ser substituídos com terapias medicamentosas (Bhatsange; Patil, 2012; Xie et al., 2014; Kopić et al., 2019).

Em seu estudo, Kopić et al. (2019), mostraram uma maior carga inflamatória utilizando interleucinas como biomarcadores, destacando que a inflamação pode agravar o quadro de insuficiência renal. Dessa maneira, há uma discussão sobre o agravamento da DRC frente à doença periodontal, inclusive Bhatsange e Patil, (2012), Xie et al. (2014), Nascimento et al. (2018) analisaram a possibilidade de uma evolução da doença periodontal ter relação com a melhora da DRC, devido à possibilidade de bacteremia transitória, que pode causar uma infecção e consequentemente uma inflamação sistêmica.

Swapna; Koppolu; Prince, (2017) salientaram que alguns fatores sistêmicos podem afetar significativamente as manifestações bucais, dentre eles, pode-se destacar o efeito da uremia que se faz presente durante o desenvolvimento da dentição e é um fator agravante para o desenvolvimento da hipoplasia de esmalte.

Além disso, a deficiência de sais como cálcio, fosfato e os altos níveis de uréia combinados com a deficiência de higiene oral podem acelerar a formação do cálculo (Martins et al., 2012; Tadakamadla et al., 2014; Xie et al., 2014; Nascimento et al., 2018). Essa mudança na quantidade de sais pode afetar a estrutura óssea resultando em osteomalácia, que resulta na perda óssea total ou parcial da lâmina dura, que será vista em exames radiográficos, os dentes então apresentam mobilidade, dessa forma, deve-se haver um cuidado especial para que não ocorra fratura óssea (Bhatsange; Patil, 2012; Gautam et al., 2014; Xie et al., 2014).

O tratamento para DRC inclui o uso de medicamentos anti-hipertensivos e a baixa ingestão de líquidos, em consequência disso, pode-se ocorrer desidratação e em função desse fato a xerostomia pode se tornar um sintoma frequente e persistente nesses pacientes (Howell; Perry; Patel, 2016; Nascimento et al., 2018; Marinosk et al., 2019; Chiu et al., 2021). Em conjunto Bhatsange; Patil, (2012), Swapna; Koppolu; Prince, (2017), Nascimento et al. (2018), Marinosk et al. (2019) sugeriram a disgeusia como um fator recorrente nesses pacientes, em consequência das toxinas urêmicas presentes nos quimiorreceptores orais em decorrência da uremia, tornando o gosto amargo e metálico.

Dentre as manifestações bucais presentes em pacientes com DRC, muitas são causadas pelo fator sistêmico urêmico, como citado por Swapna; Koppolu; Prince, (2017), Ruokonen et al. (2019) e Marinoski et al. (2019) a disgeusia é um deles, além disso, o hálito urêmico é causado pelos compósitos de amônia formados a partir da hidrólise salivar.

Andrade et al. (2015) e Silva et al. (2019) em suas pesquisas notaram que a relação da cárie com o paciente renal crônico é menor que no grupo saudável. Foram sugeridas algumas explicações para esse fato, dentre elas, o alto índice de uréia que afeta o pH bucal, uma maior quantidade de fosfato e uma baixa quantia de cálcio, afetando sua ação no processo de desmineralização e remineralização, consequentemente, aumentando a capacidade tampão exercida pela saliva, assim a doença cárie não terá uma instalação facilitada em comparação aos pacientes saudáveis (Gautam et al., 2014; Tadakamadla et al., 2014; Fregoneze et al., 2015; Swapna; Koppolu; Prince, 2017; Pham; Lee, 2018; Menezes et al., 2019; Silva et al., 2019).

$\mathrm{Na}$ literatura utilizada não foram sugeridos protocolos específicos de tratamento odontológico para esses pacientes, mas são citadas situações que ajudam a prevenir ou tratar alguns sintomas bucais. Parkar; Ajithkrishna, (2012), Fregoneze et al. (2015), Schmalz et al. (2016), sugerem que o tratamento bucal seja precoce. Swapna; Koppolu; Prince, (2017); Kopić et al. 
(2019), Menezes et al. (2019), Sharma et al. (2020), mostram em seus estudos que é necessário tratamento e acompanhamento odontológico durante toda a terapia sistêmica, englobando também a fase pré e pós transplante renal.

Durante o acompanhamento, Martins et al. (2012), Gautam et al. (2014), Xie et al. (2014), sugerem que a conscientização e o estímulo de uma boa saúde bucal é um fator decisivo durante as terapias de manutenção, tanto sistêmicas quanto locais. Durante essas consultas deve-se destacar a importância do uso do fio dental, dentifrício fluoretado e a troca constante da escova dental (Palmer et al., 2015; Nylund et al., 2017; Kesmez et al., 2020).

Além disso, Bhatsange; Patil, (2012), Nylund et al. (2017) concordam que deve ser feito a remoção de todos os focos infecciosos pré existentes ou com potencialidade de infecção. Essa remoção e manutenção periodontal pode ser feita com tratamento periodontal não cirúrgico, que inclui, profilaxias, raspagem radicular e manutenção da motivação do paciente (Andrade et al., 2015; Palmer et al., 2015; Schmalz et al., 2016; Nylund et al., 2017; Sharma., et al. 2017).

Desse modo, a literatura apesar de vasta possui concordância em relação às manifestações bucais mais prevalentes em doentes renais crônicos. Porém houve uma falta de concordância para estabelecer um protocolo de atendimento específico a esses pacientes, necessitando estudos aprofundados sobre o assunto.

\section{Conclusão}

A literatura é vasta em correlacionar a doença renal crônica com as manifestações bucais, destacando as principais disfunções, como a doença periodontal, xerostomia e disgeusia. Fatores sistêmicos causam alterações que podem afetar a cavidade bucal, logo a melhor forma de tratamento é o acompanhamento com o cirurgião dentista durante todo o curso da doença, incluindo pós-transplante.

Dessa forma é importante mais pesquisas nesse campo, principalmente relacionadas aos protocolos específicos de atendimento odontológico a esses pacientes.

\section{Referências}

Andrade, M. R. T. C., Salazar, S. L. A., Reis de Sá, L. F., Portela, M., Ferreira-Pereira, A., Soares, R. M. A., Leão, A. T. T., \& Primo, L. G. (2015). Role of saliva in the cáries experience and calculus formation of young patients undergoing hemodialysis. Clinical oral investigations, 19.

Araújo, M. V. F., Hong, B., Fava, P. L., Khan, S., Burleson, J. A., Fares, G., Samson, W., Strausbaugh, L. D., Diaz, P. I., \& Ioannidou, E. (2015). End stage renal disease as a modifier of the periodontal microbiome. BMC nephrology, 80.

Ausavarungnirun, R., Wisetsin, S., Rongkiettechakorn, N., Chaichalermsak, S., Udompol, U., \& Rattanasompattikul, M. (2016). Association of dental and periodontal disease with chronic kidney disease in patients of a single, tertiary care centre in Thailand. BMJ open, 6.

Bhatsange, A., \& Patil, S. R. (2012). Avaliação do estado de saúde periodontal em pacientes em diálise renal: estudo descritivo e transversal. Journal of Indian Society of Periodontology, 16 (1), 37-42.

Chiu, C. C., Chang, Y. C., Huang, R. Y., Chan, J. S., Chung, C. H., Chien, W. C., Kao, Y. H., \& Hsiao, P. J. (2021). Investigation of the Impact of Endodontic Therapy on Survival among Dialysis Patients in Taiwan: A Nationwide Population-Based Cohort Study. International journal of environmental research and public health, 18(1), 326.

Estrela, C. (2018). Metodologia Científica: Ciência, Ensino, Pesquisa. Editora Artes Médicas.

Fregoneze, A. P., Ortega, A. d. O. L., Brancher, J. A., Vargas, E. T., Braga, I. K., Gemelli, S., Ataide, A. F. G. d. P., Ignácio, S. A., \& Bönecker, M. J. S. (2015). Clinical evaluation of dental treatment needs in chronic renal insufficiency patients. Special care in dentistry: official publication of the American Association of Hospital Dentists, the Academy of Dentistry for the Handicapped, and the American Society for Geriatric Dentistry, 35, 63-67

Gautam, N. R., Gautam, N. S., Rao, T. H., Koganti, R., Agarwal, R., \& Alamanda, M. (2014). Effect of end-stage renal disease on oral health in patients undergoing renal dialysis: A cross-sectional study. Journal of International Society of Preventive \& Community Dentistry, 4(3), $164-169$.

Huang, R., Lin, Y., Kao, S., Shieh, Y., \& Chen, J. (2017). Dental restorative treatment expenditure and resource utilization in patients with chronic kidney disease: A nationwide population-based study. Journal of dental Science, 3, 275-282.

Kesmez, O., Frøjk, M. J., Eidemak, I., Jensen, S. B., \& Kragelund, C. (2020). Oral symptoms and pathologies in Danish patients with chronic kidney diseasea pilot study. APMIS: acta pathologica, microbiologica, et immunologica Scandinavica, 5, 401-440.

Kopić, V., Barbić, J., Petrović, S., Šahinović, I., Mihaljević, D., Kopić, A., \& Bošnjak, A. (2019). Doença periodontal em diferentes estágios da doença renal crônica. Acta clinica Croatica, 58 (4), 709-715. 
Marinoski, J. M., Bokor-Braticb, M., Miticc, I., \& Cankovic, M. (2019). Oral mucosa and salivary findings in non-diabetic patients with chronic kidney disease. Archives of oral biology, 205-211.

Martins, C., Siqueira, W. L., Oliveira, E., Nicolau, J., \& Primo, L. G. (2012). Dental calculus formation in children and adolescents undergoing hemodialysis. Pediatric nephrology (Berlin, Germany), 10, 1961-1966.

Menezes, C. R., Pereira, A. L., Ribeiro, C. C., Chaves, C. O., Guerra, R. N., Thomaz, É. B., Monteiro-Neto, V., \& Alves, C. M. (2019). Is there association between chronic kidney disease and dental cáries? A case-controlled study. Medicina oral, patologia oral y cirugia bucal, 24(2), e211-e216.

Misaki, T., Nakano, K., Ishikawa, A., Shimizu, Y., \& Fukunaga, A. (2019). Possible link between dental diseases and arteriosclerosis in patients on hemodialysis. PloS one, 12 .

Nascimento, M. A. G. D., Soares, M. S. M., Chimenos Küstner, E., Dutra, D. M., \& Cavalcanti, R. L. (2018). Oral symptoms and oral health in patients with chronic kidney disease. Revista Gaúcha de Odontologia, 2, 160-165.

Nylund, K. M., Meurman, J. H., Heikkinen, A. M., Furuholm, J. O., Ortiz, F., \& Ruokonen, H. M. (2018). Oral health in patients with renal disease: a longitudinal study from predialysis to kidney transplantation. Clinical oral investigations, 1, 339-347.

Palmer, S. C., Ruospo, M., Wong, G., Craig, J. C., Petruzzi, M., De Benedittis, M., Ford, P., Johnson, D. W., Tonelli, M., Natale, P., Saglimbene, V., Pellegrini, F., Celia, E., Gelfman, R., Leal, M. R., Torok, M., Stroumza, P., Bednarek-Skublewska, A., Dulawa, J., Frantzen, L., Ferrari, J. N., del Castillo, D., Bernat, A. G., Hegbrant, J., Wollheim, C., Gargano, L., Bots, C. P., \& Strippoli, G. F. (2015). Dental Health and Mortality in People With End-Stage Kidney Disease Treated With Hemodialysis: A Multinational Cohort Study. American journal of kidney diseases: the official journal of the National Kidney Foundation, 4, 666-676.

Parkar, S. M., \& Ajithkrishnan, CG (2012). Estado periodontal em pacientes em hemodiálise. Jornal indiano de nefrologia, 22 (4), $246-250$.

Pham, T. A. V., \& Le, D. D. (2019). Dental condition and salivary characteristics in Vietnamese patients with chronic kidney disease. International Journal of Dental Hygiene, 253-260.

Ruokonen, H., Nylund, K., Meurman, J. H., Heikkinen, A. M., Furuholm, J., Sorsa, T., Roine, R., \& Ortiz, F. (2019). Oral symptoms and oral health-related quality of life in patients with chronic kidney disease from predialysis to posttransplantation. Clinical oral investigations, 23(5), $2207-2213$.

Sarmento, D. J. d. S., Antunes, R. S. C. C. A., Cristelli, M., Braz-Silva, P. H., Maciel, R., Pestana, J. O. M. d. A., \& Gallottini, M. (2020). Oral manifestations of allograft recipients immediately before and after kidney transplantation. Acta odontologica Scandinavica, 3, $217-222$.

Schmalz, G., Kauffels, A., Kollmar , O., Slotta , J. E., Vasko, R., Müller, G. A., \& Ziebolz, R. H. e. (2016). Oral behavior, dental, periodontal and microbiological findings in patients undergoing hemodialysis and after kidney transplantation. BMC Oral Health, 16.

Sekiguchi, R. T., Pannuti, C. M., Silva Jr., H. T., Medina-Pestana, J. O., \& Romito, G. A. (2012). Decrease in oral health may be associated with length of time since beginning dialysis. Special care in dentistry: official publication of the American Association of Hospital Dentists, the Academy of Dentistry for the Handicapped, and the American Society for Geriatric Dentistry, 1, 6-10.

Sharma, L., Pradhan, D., Srivastava, R., Shukla, M., Singh, O., \& Pratik (2020). Assessment of oral health status and inflammatory markers in end stage chronic kidney disease patients: A cross-sectional study. Journal of family medicine and primary care, 9(5), 2264-2268.

Sharma, P., Cockwell, P., Dietrich, T., Ferro, C., Ives, N., \& Chapple, I. L. C. (2017). INfluence of Successful Periodontal Intervention in REnal Disease (INSPIRED): study protocol for a randomised controlled pilot clinical trial. Trials, 18, 235.

Silva, T. M. C., Alves, L. A. C., Garrido, D., Watanabe, A., Mendes, F. M., \& Ciamponi, A. L. (2019). Health and oral health?related quality of life of children and adolescents with chronic kidney disease: a cross?sectional study. Quality of life research: an international journal of quality of life aspects of treatment, care and rehabilitation, 9, 2481-2489.

Sturgill, J., Howell, S., Perry, M. M., \& Kothari, H. (2016). Protocols for treating patients with end-stage renal disease: a survey of undergraduate dental programs. Special care in dentistry: official publication of the American Association of Hospital Dentists, the Academy of Dentistry for the Handicapped, and the American Society for Geriatric Dentistry, 6, 321-326.

Swapna, L. A., Koppolu, P., \& Prince, J. (2017). Oral Health in Diabetic and Nondiabetic Patients with Chronic Kidney Disease. Saudi journal of kidney diseases and transplantation: an official publication of the Saudi Center for Organ Transplantation, Saudi Arabia, 5, 1099-1105.

Tadakamadla, J., Kumar, S., \& Mamatha, G. P. (2014). Comparative evaluation of oral health status of chronic kidney disease (CKD) patients in various stages and healthy controls. Special care in dentistry: official publication of the American Association of Hospital Dentists, the Academy of Dentistry for the Handicapped, and the American Society for Geriatric Dentistry, 3, 122-126.

Tiwari, V., Saxena, V., Bhambhal, A., Tiwari, U., Singh, A., \& Goud, S. (2013). The oral health status of patients with renal disease in central india: a preliminary study. Journal of renal care, 4, 208-213.

Xie, T., Yang, Z., Dai, G., Yan, K., Tian, Y., Zhao, D., Zou, H., Deng, F., Chen, X., \& Yuan, Q. (2014). Evaluation of the oral health status in Chinese hemodialysis patients. Hemodialysis international. International Symposium on Home Hemodialysis, 3, 668-673. 